\title{
Epiglottic Retroflexion is a Key Indicator of Functional Recovery of Post-stroke Dysphagia
}

\author{
Ji Soo Choi, $\mathrm{MD}^{1}$, Hyun Bang, $\mathrm{MD}^{1, *}$, Goo Joo Lee, $\mathrm{MD}^{1, *}$, Han Gil Seo, MD, $\mathrm{PhD}^{1}$, \\ Byung-Mo Oh, MD, $\mathrm{PhD}^{1,2}$, Tai Ryoon Han, $\mathrm{MD}, \mathrm{PhD}^{1, *}$
}

${ }^{1}$ Department of Rehabilitation Medicine, Seoul National University Hospital, Seoul National University College of Medicine, Seoul; ${ }^{2}$ National Traffic Injury Rehabilitation Hospital, Yangpyeong, Korea

Objective To evaluate the longitudinal changes of swallowing kinematics based on videofluoroscopic swallowing studies (VFSSs) in subacute stroke patients grouped according to the method of dietary intake.

Methods Sixty-nine subacute stroke patients who had taken at least 2 successive VFSSs were included. Subjects were allocated into 3 groups according to the degree of swallowing function recovery-not improved group (tube feeding recommended to patients at both studies), improved group (tube feedings recommended initially to patients and oral feeding recommended at follow-up study), and well-maintained group (oral feeding at both studies recommended to patients). Initial VFSS was performed during the subacute stage of stroke, 1 to 12 weeks after the onset of stroke, and follow-up VFSS was performed at least once. Kinematic variables were calculated by two-dimensional motion analysis of multiple structures, including the hyoid bone, epiglottis, and vocal cord. Changes of kinematic variables were analyzed in serial VFSSs.

Results At the initial VFSS, the well-maintained group showed significantly larger angles of epiglottic folding than the not improved group, while at the follow-up VFSS, the improved and the well-maintained groups showed significantly larger epiglottic folding angles than the not improved group. The distribution of epiglottic folding angles was in a dichotomous pattern, and each cluster was related to the swallowing function.

Conclusion This study showed that improved epiglottic folding angles are associated with the recovery of the swallowing process and suitability for oral feeding among various kinematic variables in subacute stroke patients.

Keywords Stroke, Deglutition, Swallowing, Epiglottis

Received July 29, 2019; Accepted September 9, 2019

Corresponding author: Byung-Mo Oh

Department of Rehabilitation Medicine, Seoul National University College of Medicine, Seoul National University Hospital, 101 Daehak-Ro, Jongnogu, Seoul 03080, Korea. Tel: +82-2-2072-2619, Fax: +82-2-6072-5244, E-mail: moya1@snu.ac.kr

${ }^{*}$ Current affiliation: Hyun Bang (Seoulhyun Rehabilitation Clinic, Seoul, Korea), Goo Joo Lee (Department of Rehabilitation, Chungbuk National University Hospital, Cheongju, Korea), Tai Ryoon Han (Department of Rehabilitation Medicine, Chungdam Hospital, Seoul, Korea)

ORCID: Ji Soo Choi (https://orcid.org/0000-0002-7242-3630); Hyun Bang (https://orcid.org/0000-0002-0502-8913); Goo Joo Lee (https://orcid. org/0000-0002-8436-4463); Han Gil Seo (https://orcid.org/0000-0001-6904-7542); Byung-Mo Oh (https://orcid.org/0000-0001-9353-7541); Tai Ryoon Han (https://orcid.org/0000-0002-7532-9068).

() This is an open-access article distributed under the terms of the Creative Commons Attribution Non-Commercial License (http://creativecommons.org/ licenses/by-nc/4.0) which permits unrestricted noncommercial use, distribution, and reproduction in any medium, provided the original work is properly cited. Copyright $\odot 2020$ by Korean Academy of Rehabilitation Medicine 


\section{INTRODUCTION}

Dysphagia after stroke is a common and disabling condition that can cause significant clinical complications such as aspiration pneumonia and malnutrition $[1,2]$. A videofluoroscopic swallowing study (VFSS) is a useful method to evaluate swallowing function in stroke patients and helps prevent aspiration pneumonia $[3,4]$. Aspiration during VFSS is an important finding related to an elevated risk of pneumonia during the subacute stage after stroke [5]. Based on the result of a VFSS, physicians can modify diets for the patients, educate compensatory swallowing techniques [6], and decide whether to remove nasogastric tubes [7].

Many studies have investigated VFSS images with kinematic analysis in normal subjects and stroke patients to determine the mechanisms of normal swallowing and the pathophysiology associated with dysphagia [8-11]. One of our previous studies demonstrated that delayed triggering of the swallowing reflex is a characteristic finding in stroke patients with aspiration [12]. It also showed that the distribution of the epiglottic tilt angle has a dichotomous pattern, which implies that epiglottic dysfunction in stroke patients occurs in an all-or-nothing manner. These findings were reproduced in another of our previous studies, which showed that decreased epiglottic tilting in post-stroke dysphagia is highly associated with the risk of aspiration [13]. These studies focused on investigating the pathophysiology of dysphagia with a special interest on aspiration. However, none of these studies related the kinematic data to actual diet recommendations made from the VFSS.

This study aimed to evaluate the longitudinal changes of the swallowing process with a kinematic analysis of VFSS in subacute stroke patients grouped by types of dietary intake method recommendations from VFSSs. It is important in clinical practice to wean off tube feedings since prolonged nasogastric tube feedings are harmful to the swallowing function $[14,15]$. Therefore, we aimed to find kinematic variables associated with the recovery from dysphagia and those suitable for oral feeding.

\section{MATERIALS AND METHODS}

\section{Participants}

In this retrospective study, we reviewed the video files and reports of VFSSs which were performed between January 1, 2005 and February 28, 2011 in our hospital. Stroke patients who were diagnosed by neurologists with confirmatory computed topography or magnetic resonance image findings were our primary interest. Among them, patients who met all the following inclusion criteria were included: (1) initial VFSSs performed during the subacute stage from 1 week to 12 weeks after the onset of stroke; (2) follow-up VFSSs performed at least once after the initial VFSS; and (3) both studies included the test for swallowing $2 \mathrm{~mL}$ of thin liquid. Those with a previous history of stroke, Parkinson disease, brain tumors, head and neck cancers, or any other disease which could cause dysphagia were excluded. We reviewed the medical records of patients included in this study for clinical information, including demographic data, location and type of lesions, the dates of the studies, and the onset of the strokes. After screening 693 stroke patients, 69 patients were included. This study was approved by the Institutional Review Board of Seoul National University Hospital (No. 0807-010-249).

We classified every patient included in this study into 3 groups using the following criteria: (1) not improved group, if initial and follow-up VFSS both recommended tube feeding to the patient; (2) improved group, if initial VFSS recommended tube feeding and follow-up VFSS recommended oral feeding to the patient; and (3) wellmaintained group, if initial and follow-up VFSS both recommended oral feeding to the patient. All patients followed the recommendations of the VFSS to start tube feedings or diet modifications, and began swallowing rehabilitation therapy after their initial VFSS. Swallowing rehabilitation was guided by specialized occupational therapists for 30 minutes per every working day during inpatient stays. If indicated, swallowing rehabilitation was continued after discharge at a weekly outpatient session. Swallowing rehabilitation included compensatory maneuver training, oropharyngeal exercises, pharyngeal thermos-tactile stimulation, and laryngeal electrical stimulation.

\section{Videofluoroscopic swallowing study}

VFSS was performed with a modified Logemann protocol [16], as described in previous studies [12,13]. Patients were seated on a chair and lateral images were recorded by a fluoroscope. Nasogastric tubes, if present, were re- 
moved before the onset of the VFSS. Several test diets including $2 \mathrm{~mL}$ and $5 \mathrm{~mL}$ of diluted barium (35\% weight), pudding, curd-type yogurt, and boiled rice were prepared for each patient. The study was performed by at least 2 experienced physiatrists, and they decided which diets to test based on the patient's VFSS findings. A 24-mm diameter coin was placed on the patient's chin as a reference for length measurements. All video images were recorded at a resolution of $720 \times 480$ pixels and at a speed of 30 frames per second. Digital image files were acquired by a frame grabber board (Pinnacle Studio MovieBox DV; Pinnacle System Inc., Mountain View, CA, USA) and image processing software (Pinnacle Studio 9.0; Pinnacle System Inc.). For optimal imaging of pharyngeal soft tissues, the $\mathrm{X}$-ray voltage was set at a $40-\mathrm{kV}$ peak.

The images were analyzed by the physiatrists who performed the study. Based on the findings of the VFSS and clinical information regarding each patient, the study report was made by a consensus of the physiatrists. The American Speech-Language-Hearing Association National Outcome Measurement System (ASHA NOMS) swallowing scale was included in the reports and was used to determine whether tube feedings should be recommended (ASHA NOMS score $<4$ ). The Videofluoroscopic Dysphagia Scale (VDS) [17] was also reported in all studies.

\section{Kinematic analysis}

We analyzed the VFSS images of the first trial of swallowing $2 \mathrm{~mL}$ of diluted barium using a previously described method [10]. Locations of the anterior-superior border of the hyoid bone and vocal cord and base-to-tip margins of the epiglottis in each frame were acquired using kinematic analysis software (Ariel Performance Analysis System; Ariel Dynamics Inc., Trabuco Canyon, CA, USA). A zero-point of coordinates was set at the anterior border of the inferior endplate of the $\mathrm{C} 4$ vertebrae. The line connecting the zero-point and the anterior margin of the inferior endplate of the $\mathrm{C} 2$ vertebrae was defined as the $y$-axis, and the line perpendicular to the y-axis and passing through the zero-point was defined as the $\mathrm{x}$-axis. The kinematic data were converted to an actual length scale using the $24-\mathrm{mm}$ diameter coin as the reference. For epiglottic retroflexion or folding, the initial angle was defined as $0^{\circ}$. From the kinematic data of both the initial and follow-up VFSSs for each patient, the maximum displacement (in $\mathrm{mm}$ ) of the hyoid bone and the vocal cord in both vertical and horizontal directions, and the epiglottic folding angle $\left(^{\circ}\right)$ were measured. MATLAB 7.4 (MathWorks Inc., Natick, MA, USA) was used for all measurements.

\section{Statistical analysis}

The 3 groups were compared for patient characteristics, VDS, and kinematic variables. All continuous variables were tested for normality using the Shapiro-Wilk test. One-way analysis of variance (ANOVA), chi-square $\left(\chi^{2}\right)$ tests, Fisher exact tests, or Kruskal-Wallis tests were used for intergroup comparisons. If significant differences were noted in a variable, we performed post-hoc tests using the Bonferroni correction, and plotted histograms and a scatter plot for that variable. We also performed a Spearman rank correlation between the noted variable and ASHA NOMS swallowing scores.

Since epiglottic folding angles were dichotomously dis-

Table 1. Patients' characteristics $(n=69)$

\begin{tabular}{lc}
\hline \multicolumn{1}{c}{ Characteristic } & Value \\
\hline Age (yr) & $67.0 \pm 12.8$ \\
Sex & $43(62)$ \\
$\quad$ Male & $26(38)$ \\
\hline Female & $14(20)$ \\
\hline Grouping as the changes of recommended diet \\
\hline Not improved group & $21(30)$ \\
\hline Improved group & $34(49)$ \\
\hline Well-maintained group & \\
Type of stroke & $51(74)$ \\
\hline Ischemic & $18(26)$ \\
\hline Hemorrhagic & \\
\hline Location of lesion & $48(70)$ \\
\hline Supratentorial & $21(30)$ \\
\hline Infratentorial & \\
\hline Side of lesion in the brain & $37(54)$ \\
\hline Right side & $25(36)$ \\
\hline Left side & $7(10)$ \\
\hline Bilateral & $26.6 \pm 12.7$ \\
\hline Post-stroke day at the initial VFSS & $76.9 \pm 60.6$ \\
\hline Post-stroke day at the follow-up VFSS & $50.3 \pm 59.7$ \\
\hline Interval between the 2 studies (day)
\end{tabular}

Values are mean \pm standard deviation and number (\%). VFSS, videofluoroscopic swallowing study. 
tributed in our previous studies [12,13], we aimed to determine a cutoff value of epiglottic folding angles for oral feeding suitability (ASHA NOMS $\geq 4$ ) by using epiglottic folding angle data from the well-maintained group and the follow-up study of the improved group, in which all patients were suitable for oral feeding. We used the interquartile rule to remove outliers by subtracting 1.5 times of interquartile range from the first quartile [18], and assigned that value as the cutoff point.

The changes of the kinematic variables between the initial and follow-up studies were tested by paired t-tests or Wilcoxon signed rank tests. SPSS version 22.0 software (SPSS Inc., Chicago, IL, USA) was used for all statistical analyses. Statistical significance was defined as a p-value $<0.05$.

\section{RESULTS}

\section{Patient characteristics}

A total of 69 patients were included for analysis. Demographic data and other clinical characteristics of the patients included in this study are summarized in Table 1. None of the patients experienced neurological deteriora- tion between the initial and follow-up VFSSs.

The results of comparing patient characteristics among the 3 groups are summarized in Table 2. There were no significant differences in patient characteristics among the 3 groups except sex distributions and laterality of the lesions $\left(\chi^{2}=7.38, p=0.03\right.$ by $\chi^{2}$ test for sex; $p=0.04$ by Fisher exact test for laterality). Post-hoc analysis of sex revealed that there was a difference between the not improved and well-maintained groups with borderline significance ( $\chi^{2}=6.10, p=0.05$ by $\chi^{2}$ test with Bonferroni correction), but no difference between the other pairs. Post-hoc analysis of the laterality of lesions failed to reveal significant differences in all 3 pairs.

\section{Comparisons of kinematic variables}

The results of comparing kinematic variables among the 3 groups are summarized in Table 3 . In both the initial and follow-up studies, VDS was different among the 3 groups $(H=20.40, p<0.001$ in the initial study; $H=28.32$, $\mathrm{p}<0.001$ in the follow-up study) using the Kruskal-Wallis test. The well-maintained group showed a lower VDS than the other 2 groups in the initial study $(U=68.5$, $p<0.001$ for the not improved group; $U=154, p=0.001$

Table 2. Comparisons of patient characteristics among 3 groups

\begin{tabular}{|c|c|c|c|c|}
\hline & Not improved $(n=14)$ & Improved $(\mathrm{n}=21)$ & Well-maintained $(n=34)$ & p-value \\
\hline Ages (yr) & $65.0 \pm 8.3$ & $64.1 \pm 15.4$ & $69.6 \pm 12.4$ & $0.24^{\mathrm{a})}$ \\
\hline Sex & & & & $0.03^{\mathrm{b})}$ \\
\hline Male & $12(86)$ & $15(71)$ & $16(47)$ & \\
\hline Female & $2(14)$ & $6(29)$ & $18(53)$ & \\
\hline Type of stroke & & & & $0.23^{\mathrm{c})}$ \\
\hline Ischemic & $10(71)$ & $13(62)$ & $28(82)$ & \\
\hline Hemorrhagic & $4(29)$ & $8(38)$ & $6(18)$ & \\
\hline Location of lesion & & & & $0.06^{\mathrm{c})}$ \\
\hline Supratentorial & $6(43)$ & $17(81)$ & $25(74)$ & \\
\hline Infratentorial & $8(57)$ & $4(19)$ & $9(26)$ & \\
\hline Side of lesion in the brain & & & & $0.04^{\mathrm{c})}$ \\
\hline Right side & $7(50)$ & $9(43)$ & $21(62)$ & \\
\hline Left side & $4(29)$ & $8(38)$ & $13(38)$ & \\
\hline Bilateral & $3(21)$ & $4(19)$ & $0(0)$ & \\
\hline Post-stroke day at the initial VFSS & $29.0 \pm 15.1$ & $28.1 \pm 16.5$ & $28.4 \pm 12.9$ & $0.41^{\mathrm{d})}$ \\
\hline Post-stroke day at the follow-up VFSS & $67.2 \pm 53.5$ & $126.1 \pm 111.1$ & $117.7 \pm 97.8$ & $0.11^{\mathrm{d})}$ \\
\hline Interval between the 2 studies (day) & $38.2 \pm 49.8$ & $98.0 \pm 106.3$ & $89.3 \pm 96.1$ & $0.55^{\mathrm{d})}$ \\
\hline
\end{tabular}

Values are mean \pm standard deviation and number (\%).

VFSS, videofluoroscopic swallowing study.

${ }^{\text {a) }}$ One-way analysis of variance, ${ }^{\text {b) }}$ chi-square test, ${ }^{\text {c) }}$ Fisher exact test, ${ }^{\text {d) }}$ Kruskal-Wallis test. 
Table 3. Kinematic variables and global assessment of videofluoroscopic swallowing studies

\begin{tabular}{|c|c|c|c|c|}
\hline & Not improved & Improved & Well-maintained & p-value \\
\hline \multicolumn{5}{|l|}{ Initial study } \\
\hline VDS & $42.89 \pm 13.70$ & $40.45 \pm 15.45$ & $24.37 \pm 11.63^{* *}$ & $<0.001^{\text {a) }}$ \\
\hline $\mathrm{EA}\left({ }^{\circ}\right)$ & $45.03 \pm 27.07$ & $76.10 \pm 41.41$ & $94.28 \pm 19.04^{*}$ & $0.001^{\text {a) }}$ \\
\hline MaxHH (mm) & $10.99 \pm 3.44$ & $10.01 \pm 3.29$ & $9.32 \pm 6.30$ & $0.58^{\mathrm{b})}$ \\
\hline $\operatorname{MaxHV}(\mathrm{mm})$ & $15.04 \pm 4.93$ & $13.53 \pm 6.05$ & $11.56 \pm 4.31$ & $0.08^{\mathrm{a})}$ \\
\hline MaxVH (mm) & $5.75 \pm 1.52$ & $5.20 \pm 2.46$ & $5.95 \pm 2.86$ & $0.42^{\mathrm{a})}$ \\
\hline $\operatorname{MaxVV}(\mathrm{mm})$ & $22.88 \pm 5.26$ & $20.85 \pm 6.82$ & $21.58 \pm 6.09$ & $0.64^{\mathrm{b})}$ \\
\hline \multicolumn{5}{|l|}{ Follow-up study } \\
\hline VDS & $51.36 \pm 12.09$ & $29.81 \pm 10.94^{*}$ & $21.62 \pm 13.18^{* *}$ & $<0.001^{a)}$ \\
\hline $\mathrm{EA}\left({ }^{\circ}\right)$ & $46.97 \pm 26.38$ & $90.25 \pm 37.20^{*}$ & $95.83 \pm 19.77^{*}$ & $<0.001^{\text {a) }}$ \\
\hline MaxHH (mm) & $9.98 \pm 2.43$ & $10.58 \pm 3.54$ & $8.73 \pm 6.13$ & $0.37^{\mathrm{b})}$ \\
\hline $\operatorname{MaxHV}(\mathrm{mm})$ & $14.76 \pm 6.97$ & $14.14 \pm 4.54$ & $11.42 \pm 5.21$ & $0.07^{\mathrm{a})}$ \\
\hline MaxVH (mm) & $5.11 \pm 2.25$ & $7.03 \pm 3.37$ & $5.83 \pm 2.49$ & $0.21^{\mathrm{a})}$ \\
\hline $\operatorname{MaxVV}(\mathrm{mm})$ & $21.57 \pm 7.12$ & $21.57 \pm 5.25$ & $20.89 \pm 6.73$ & $0.91^{\mathrm{b})}$ \\
\hline
\end{tabular}

Values are mean \pm standard deviation.

VDS, Videofluoroscopic Dysphagia Scale; EA, epiglottic folding angle; MaxHH, maximal horizontal movement of hyoid bone; MaxHV, maximal vertical movement of hyoid bone; MaxVH, maximal horizontal movement of vocal cord; MaxVV, maximal vertical movement of vocal cord.

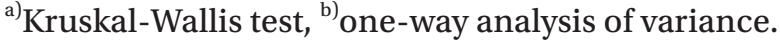

*p $<0.05$, compared with "not improved" group (by post-hoc analysis with Bonferroni correction).

**p $<0.05$, compared with "not improved" and "improved" groups (by post-hoc analysis with Bonferroni correction).

for the improved group) using the Mann-Whitney test with Bonferroni correction, as well as in the followup study $(\mathrm{U}=31, \mathrm{p}<0.001$ for the not improved group; $\mathrm{U}=216, \mathrm{p}=0.045$ for the improved group) using the MannWhitney test with Bonferroni correction. At the followup study, the VDS of the improved group was lower than that of the not improved group $(U=23, p<0.001)$ using the Mann-Whitney test with Bonferroni correction.

In both studies, the angle of epiglottic folding was the only kinematic variable that showed a significant difference among the 3 groups $(H=13.74, p=0.001$ in the initial study; $\mathrm{H}=18.69, \mathrm{p}<0.001$ in the follow-up study) using the Kruskal-Wallis test. In initial VFSSs, post-hoc analysis revealed that the epiglottic folding angle of the wellmaintained group was significantly higher than that of the not improved group ( $U=55, p<0.001)$ using the MannWhitney test with Bonferroni correction (Fig. 1). In the follow-up VFSS, post-hoc analysis revealed that the epiglottic folding angles of the improved and well-maintained groups were both significantly higher than that of the not improved group ( $U=57, p=0.006$ between the not improved group and improved group; $U=39, p<0.001$ between the not improved group and well-maintained group) using the by Mann-Whitney test with Bonferroni correction (Fig. 1). Within-subject comparisons of kinematic variables between initial and follow-up VFSSs revealed no significant differences in any variables.

\section{Change of epiglottic folding angle}

The histograms of epiglottic folding angles in initial and follow-up VFSSs showed a definite dichotomous pattern (Fig. 2). We created a scatter plot of epiglottic folding angles of the 2 studies (Fig. 3), and the graph showed that the distribution of the epiglottic folding angle was different between the groups. The not improved group patients primarily clustered in the lower epiglottic folding angle while the well-maintained group patients clustered in the higher folding angle. The improved group patients were distributed in both clusters, primarily in the higher cluster at the follow-up study. We determined the cutoff value of epiglottic folding angles for oral feeding suitability from the studies in which oral feedings were suitable for all patients (Fig. 2C). After subtracting 1.5 times the interquartile range from the first quartile to remove out- 
liers, we found the cutoff value to be $51.41^{\circ}$ (first quantile $=84.69^{\circ}$, interquartile range $=22.19^{\circ}$ ).

We added vertical and horizontal auxiliary lines at $51.41^{\circ}$ in Fig. 3. Nine of 14 patients in the not improved

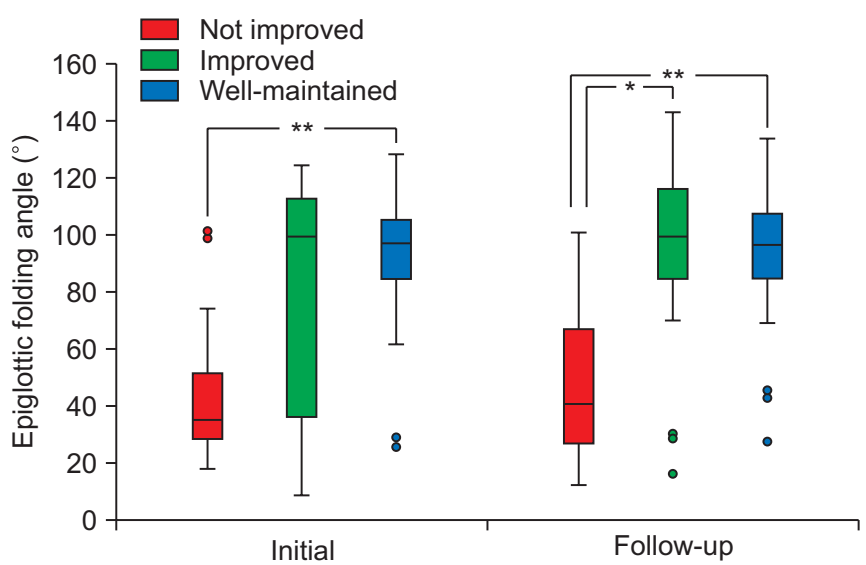

Fig. 1. Comparison of epiglottic folding angle between 3 groups at initial and follow-up studies. ${ }^{*} \mathrm{p}<0.05,{ }^{* *} \mathrm{p}<0.001$ in Mann-Whitney test with Bonferroni correction.

(A)

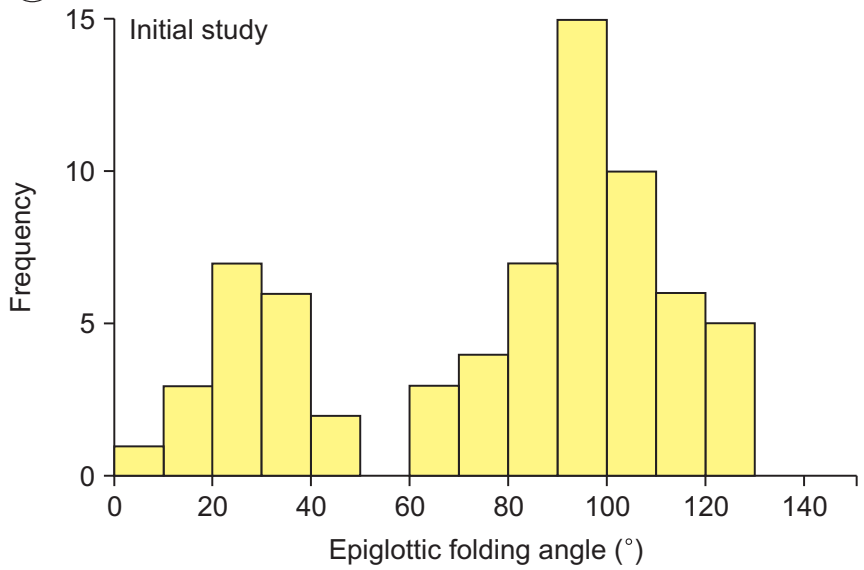

(C)

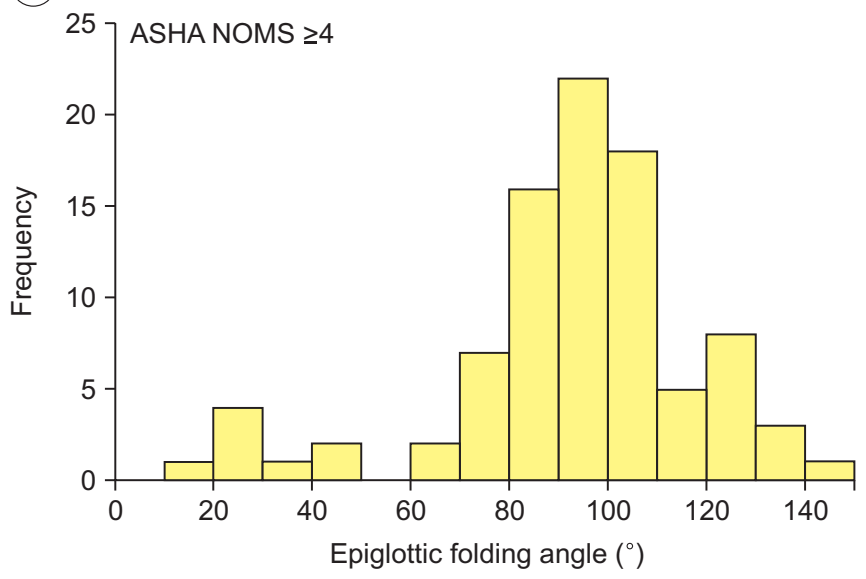

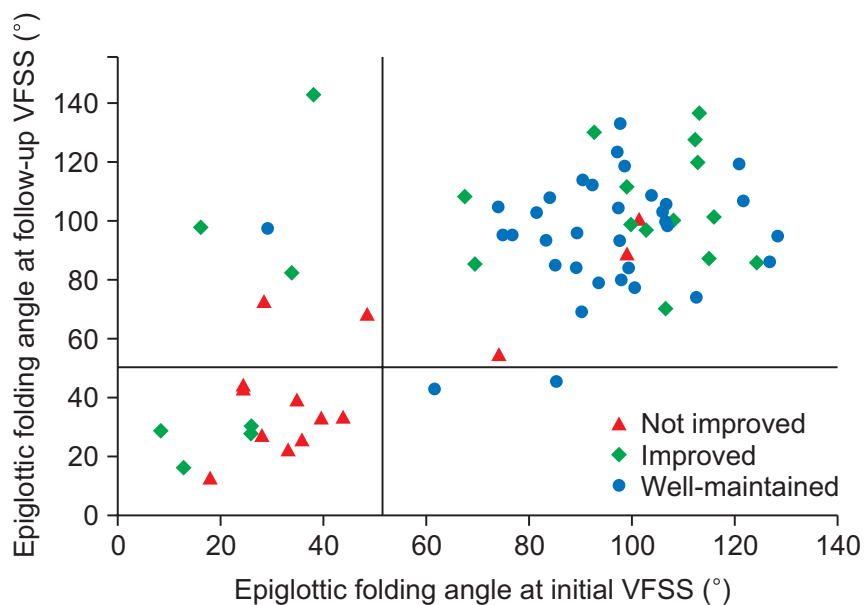

Fig. 3. A scatter plot of the epiglottic folding angle at initial and follow-up studies. Vertical and horizontal auxiliary lines are at $51.41^{\circ}$. Nine out of 14 patients in "not improved" group have low angles of epiglottic folding, while 31 out of 34 patients of "well-maintained" group have high angles of epiglottic folding in both studies.

(B)

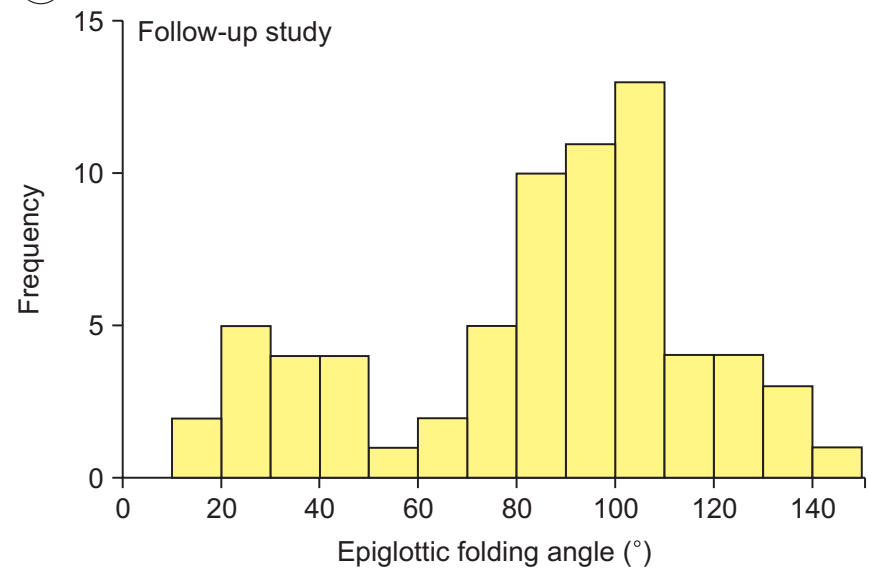

Fig. 2. Distribution of epiglottic folding angle at (A) initial study and (B) follow-up study. Both histograms show a definite dichotomous pattern of distribution. (C) Data from follow-up study of "improved" group and both studies of "well-maintained" group are gathered to show the distribution of epiglottic folding angle among patients suitable for oral feeding. 
group had low angles for both studies, while 31 of 34 patients in the well-maintained group had high angles for both studies. For patients in the improved group, we created 3 subgroups based on the change of the epiglottic folding angle: subgroup 1 for patients who showed an epiglottic folding angle $<51.41^{\circ}$ at both initial and follow-up VFSSs $(\mathrm{n}=4)$; subgroup 2 for patients who showed an epiglottic folding angle $<51.41^{\circ}$ initially, but later showed a higher range angle $(\mathrm{n}=3)$; and subgroup 3 for patients who showed an epiglottic folding angle $>51.41^{\circ}$ at the initial study $(\mathrm{n}=14)$. We plotted the ASHA NOMS swallowing scale of the patients in each subgroup at initial and follow-up VFSSs (Fig. 4). The mean ASHA NOMS swallowing scores at initial VFSS were: $1.0 \pm 0.0$ in subgroups 1 and 2, and $1.7 \pm 0.7$ in subgroup $3(\mathrm{H}=5.90$, $\mathrm{p}=0.05)$ using the Kruskal-Wallis test; and at follow-up VFSS, the scores were $4.8 \pm 0.5$ in subgroup $1,4.7 \pm 0.6$ in subgroup 2, and $5.5 \pm 0.7$ in subgroup $3(\mathrm{H}=6.26, \mathrm{p}=0.04)$ using the Kruskal-Wallis test. As the angle of epiglottic folding improved, the ASHA NOMS swallowing scale results also improved, which indicated fewer limitations

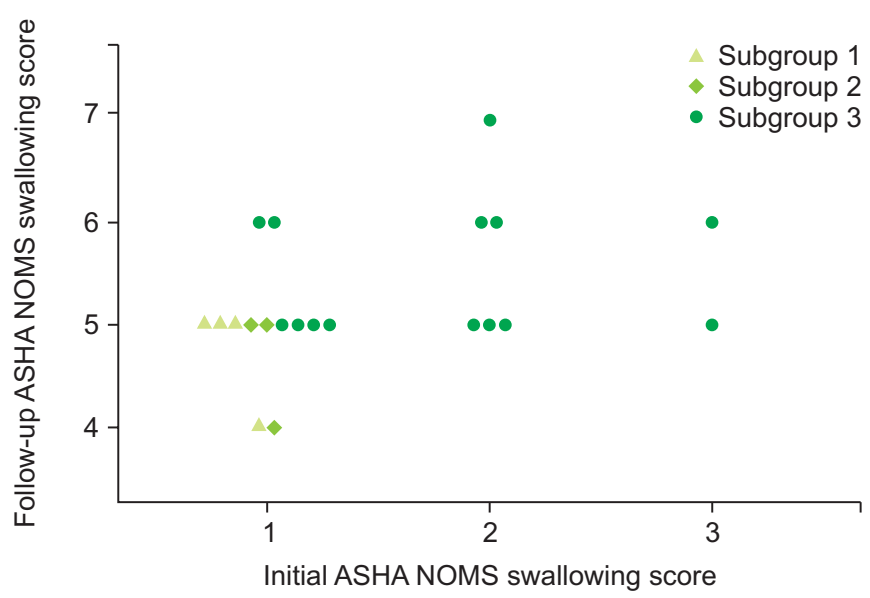

Fig. 4. Change of diet in "improved" group patients between the initial and follow-up studies. Based on the change of epiglottic folding angle, the patients are grouped into 3 subgroups: subgroup 1, epiglottic folding angles of the 2 studies are lower than $51.41^{\circ}$; subgroup 2, epiglottic folding angle is lower than $51.41^{\circ}$ in the initial study, but improved to higher range in follow-up study; subgroup 3, epiglottic folding angle is higher than $51.41^{\circ}$ at the initial study. It shows that the higher epiglottic folding angle is associated with less limitation on a diet. ASHA NOMS, American Speech-Language-Hearing Association National Outcome Measurement System. for oral diets. Spearman correlation analysis also showed a significant correlation between the epiglottic folding angle and ASHA NOMS swallowing score in both studies $\left(r_{s}=0.353, p=0.003\right.$ at the initial study; $r_{s}=0.367, p=0.002$ at the follow-up study).

\section{DISCUSSION}

In this retrospective study, VDS and angle of epiglottic folding were the only variables from the VFSS that were related to oral feeding suitability. Epiglottic folding angles were dichotomously distributed, and higher degrees of angles were significantly associated with good swallowing function. Unlike previous studies using kinematic analysis that only assessed the findings of aspiration or penetration $[10,12,13]$, we discovered a significant relationship between the functional level of oral intake and the movement of the epiglottis which was a novel finding of the current study. Clinical decisions, such as diet recommendations, are made from the overall results of VFSSs and are not solely dependent on the findings of aspirations of thin liquid [6]. Thus, there is a gap between the knowledge from previous kinematic studies and actual clinical practice. During VFSS, we used not only thin liquid, but also other test intakes such as yogurt, nectar, and solid food, with different volumes and viscosities [16]. Although we did not analyze the hyolaryngeal kinematics of all types of boluses in this study, which are known to be altered by each type of bolus [19-21], diet recommendations were made from findings of all of these different test diets. Based on those diet recommendations, we grouped the patients and compared kinematics, and discovered that the only significant kinematic variable was the epiglottic folding angle which appears to be the most relevant factor for the actual swallowing function in stroke patients.

In a previous study of 28 stroke patients with aspiration at the initial VFSS, the maximal epiglottic tilt angle of those who recovered from aspiration was higher than those who did not, with borderline significance [12]. In another study with a larger sample size $(n=68)$, stroke patients with aspirations had significantly smaller epiglottic tilt angles than those without aspirations [13]. In the present study, the well-maintained group, for whom oral feedings were recommended, had a significantly larger angle of epiglottic folding than the other groups at initial 
VFSS. The improved group patients whose recommendations included weaning off tube feeding at the followup VFSSs, showed improved epiglottic folding angles compared to the not improved group patients. All these findings suggest that sufficient epiglottic folding may be necessary for safe oral feeding in stroke patients. Epiglottic folding for airway protection is associated with anterior displacement of the hyoid bone and laryngeal elevation with upper esophageal sphincter relaxation [2224]. Thus, reduced epiglottic folding may result from impaired pharyngeal movement causing aspiration and lower ASHA NOMS swallowing scores.

The distribution of epiglottic folding angles followed a dichotomous pattern, similar to our previous 2 studies [12,13], with relevance to diet recommendations. The dichotomous distribution implies that epiglottic folding decreases in an all-or-nothing manner in stroke patients. The first epiglottic movement to reach a horizontal level is mainly achieved by thyrohyoid approximation, and the second epiglottic movement of downfolding below the horizontal level occurs by anterior displacement of the hyoid bone, bolus movement, tongue base retraction, and intrapharyngeal pressure gradients [22-24]. It is our opinion that reaching a horizontal level in the first epiglottic movement is a threshold for the second epiglottic movement. Therefore, the patients whose thyrohyoid approximations are impaired by stroke may show distinctively insufficient epiglottic movement due to failure to reach horizontal levels. To confirm our hypothesis, further studies using kinematic analyses of thyrohyoid approximations would be necessary.

Among dichotomously distributed epiglottic folding angles, the not improved group patients clustered primarily at the lower epiglottic folding angle while the wellmaintained group patients clustered at higher epiglottic folding angles (Fig. 3). The improved group patients were distributed in both clusters with more than half having an epiglottic folding angle in the higher range, and tended to have less limited diet recommendations at follow-up studies. Correlation analysis also confirmed this tendency. This implies that if the initial epiglottic folding angles were preserved, patients would most likely eventually achieve oral feedings with fewer limitations.

The VDS comprises 14 items regarding VFSS findings and predicts the long-term prognosis of post-stroke dysphagia with moderate inter-rater reliability $[17,25]$. It also significantly correlates with ASHA NOMS swallowing scores in various etiologies of dysphagia [26]. Our results also support the validity of VDS as a quantitative scale of dysphagia. The VDS represents oral and pharyngeal functions, which include adequate tongue movement and retraction, prompt pharyngeal triggering, low amount of pharyngeal residues, and complete airway protection [17]. Since clinicians decide diet recommendations based on all of these findings, it is not surprising that the VDS is well correlated with diet recommendations. An interesting result was that epiglottic folding angles, which are a simple variable focused on a single structure, are also good indicators of the swallowing function.

Patient characteristics were heterogeneous among the participants in this retrospective study. The sex ratio was uneven overall, especially between the not improved group and the well-maintained group. This implies that the male gender is more likely to receive recommendations for tube feeding. This is similar to previous studies that reported that the male gender was associated with post-stroke dysphagia, pneumonia, and feeding tube placement [27-29]. Our patients with bilateral hemisphere involvement were more likely to be in the not improved group ( 3 of 7 ) and none of them were in the well-maintained group. This implies that bilateral lesions would be a predictor for persistent dysphagia, as previously noted [30]. It would be interesting to perform subgroup analysis for kinematics for different patient characteristics, but this was beyond the scope of this small sample size retrospective study.

There are several limitations in this study. First, the timing of initial VFSSs and follow-up periods were heterogeneous, which made it impossible to analyze the effect of recovery time. However, since there was no significant difference of post-stroke time and study intervals among the 3 groups, it would not have distorted the interpretation of our data. Secondly, we only analyzed the kinematics of the pharyngeal phase of the first swallow of thin liquid. However, when a patient takes a meal, a number of swallows of different types of food occur. There would be different kinematics between the test swallow in the VFSS and the numerous swallows in real-world situations. Additionally, the movements of oropharyngeal structures during the oral and esophageal phases are important, but were not included in our analysis. Thirdly, we did not include temporal variables which are known to be 
characteristically delayed in stroke patients and related to aspiration $[12,13]$. Fourthly, the uneven distribution of patients across the 3 groups could be a limiting factor in this study. Lastly, the sample size was small and might reduce statistical power.

In conclusion, this study suggests that higher epiglottic folding angles may be associated with swallowing function recovery and suitability for oral feedings among various kinematic variables in subacute stroke patients. Further well-controlled prospective studies to analyze the kinematic and temporal variables with different types of boluses would provide us with additional knowledge of the pathophysiology and outcomes of post-stroke dysphagia and would create a reference for systematic diet prescriptions for post-stroke dysphagia. Therapeutic interventions on suprahyoid structures to reach the threshold of epiglottic folding would be another future task aimed at improving oral feeding suitability.

\section{CONFLICT OF INTEREST}

No potential conflict of interest relevant to this article was reported.

\section{AUTHOR CONTRIBUTION}

Conceptualization: Bang H, Lee GJ, Seo HG, Oh BM, Han TR. Methodology: Bang H, Lee GJ, Seo HG, Oh BM, Han TR. Formal analysis: Choi JS, Bang H, Lee GJ, Seo HG, Oh BM, Han TR. Project administration: Bang H, Lee GJ, Oh BM. Visualization: Choi JS, Bang H, Lee GJ. Writing - original draft: Choi JS. Writing - review and editing: Choi JS, Bang H, Lee GJ, Seo HG, Oh BM. Approval of final manuscript: all authors.

\section{REFERENCES}

1. Martino R, Foley N, Bhogal S, Diamant N, Speechley M, Teasell R. Dysphagia after stroke: incidence, diagnosis, and pulmonary complications. Stroke 2005;36:2756-63.

2. Foley NC, Martin RE, Salter KL, Teasell RW. A review of the relationship between dysphagia and malnutrition following stroke. J Rehabil Med 2009;41:707-13.

3. Daniels SK, Brailey K, Priestly DH, Herrington LR, Weisberg LA, Foundas AL. Aspiration in patients with acute stroke. Arch Phys Med Rehabil 1998;79:14-9.

4. Kim SB, Lee SJ, Lee KW, Lee JH, Kim DW. Usefulness of early videofluoroscopic swallowing study in acute stroke patients with dysphagia. Ann Rehabil Med 2018;42:42-51.

5. Holas MA, DePippo KL, Reding MJ. Aspiration and relative risk of medical complications following stroke. Arch Neurol 1994;51:1051-3.

6. Martin-Harris B, Logemann JA, McMahon S, Schleicher M, Sandidge J. Clinical utility of the modified barium swallow. Dysphagia 2000;15:136-41.

7. Hwang JM, Cheong YS, Kang MG, Chun SM, Min YS, Lee YS, et al. Recommendation of nasogastric tube removal in acute stroke patients based on videofluoroscopic swallow study. Ann Rehabil Med 2017;41:915.

8. Kim Y, McCullough GH, Asp CW. Temporal measurements of pharyngeal swallowing in normal populations. Dysphagia 2005;20:290-6.

9. Logemann JA, Pauloski BR, Rademaker AW, Colangelo LA, Kahrilas PJ, Smith CH. Temporal and biomechanical characteristics of oropharyngeal swallow in younger and older men. J Speech Lang Hear Res 2000;43:1264-74.

10. Paik NJ, Kim SJ, Lee HJ, Jeon JY, Lim JY, Han TR. Movement of the hyoid bone and the epiglottis during swallowing in patients with dysphagia from different etiologies. J Electromyogr Kinesiol 2008;18:329-35.

11. Kim Y, McCullough GH. Maximal hyoid excursion in poststroke patients. Dysphagia 2010;25:20-5.

12. Seo HG, Oh BM, Han TR. Longitudinal changes of the swallowing process in subacute stroke patients with aspiration. Dysphagia 2011;26:41-8.

13. Seo HG, Oh BM, Han TR. Swallowing kinematics and factors associated with laryngeal penetration and aspiration in stroke survivors with dysphagia. Dysphagia 2016;31:160-8.

14. Kwak HJ, Kim L, Ryu BJ, Kim YH, Park SW, Cho DG, et al. Influence of nasogastric tubes on swallowing in stroke patients: measuring hyoid bone movement with ultrasonography. Ann Rehabil Med 2018;42:5519.

15. Wang ZY, Chen JM, Ni GX. Effect of an indwelling nasogastric tube on swallowing function in elderly poststroke dysphagia patients with long-term nasal feeding. BMC Neurol 2019;19:83. 
16. Logemann JA. Manual for the videofluorographic study of swallowing. Austin, TX: Pro-ED; 1993.

17. Han TR, Paik NJ, Park JW, Kwon BS. The prediction of persistent dysphagia beyond six months after stroke. Dysphagia 2008;23:59-64.

18. Walfish S. A review of statistical outlier methods. Pharm Technol 2006;30:82-86.

19. Choi KH, Ryu JS, Kim MY, Kang JY, Yoo SD. Kinematic analysis of dysphagia: significant parameters of aspiration related to bolus viscosity. Dysphagia 2011;26:392-8.

20. Nagy A, Molfenter SM, Peladeau-Pigeon M, Stokely S, Steele CM. The effect of bolus volume on hyoid kinematics in healthy swallowing. Biomed Res Int 2014;2014:738971.

21. Lenell C, Brates D, Pearson WG Jr, Molfenter S. Variations in healthy swallowing mechanics during various bolus conditions using computational analysis of swallowing mechanics (CASM). Dysphagia 2019 Jun 4 [Epub]. http://doi.org/10.1007/s00455-019-10026-9.

22. Logemann JA, Kahrilas PJ, Cheng J, Pauloski BR, Gibbons PJ, Rademaker AW, et al. Closure mechanisms of laryngeal vestibule during swallow. Am J Physiol 1992;262:G338-44.

23. Vandaele DJ, Perlman AL, Cassell MD. Intrinsic fibre architecture and attachments of the human epiglottis and their contributions to the mechanism of deglutition. J Anat 1995;186:1-15.

24. Matsuo K, Palmer JB. Anatomy and physiology of feeding and swallowing: normal and abnormal. Phys Med Rehabil Clin N Am 2008;19:691-707.

25. Kim DH, Choi KH, Kim HM, Koo JH, Kim BR, Kim TW, et al. Inter-rater reliability of videofluoroscopic dysphagia scale. Ann Rehabil Med 2012;36:791-6.

26. Kim J, Oh BM, Kim JY, Lee GJ, Lee SA, Han TR. Validation of the videofluoroscopic dysphagia scale in various etiologies. Dysphagia 2014;29:438-43.

27. Henke C, Foerch C, Lapa S. Early screening parameters for dysphagia in acute ischemic stroke. Cerebrovasc Dis 2017;44:285-90.

28. Brown K, Cai C, Barreto A, Shoemaker P, Woellner J, $\mathrm{Vu} \mathrm{K}$, et al. Predictors of percutaneous endoscopic gastrostomy placement in acute ischemic stroke. J Stroke Cerebrovasc Dis 2018;27:3200-7.

29. Beharry A, Michel P, Faouzi M, Kuntzer T, Schweizer V, Diserens K. Predictive factors of swallowing disorders and bronchopneumonia in acute ischemic stroke. J Stroke Cerebrovasc Dis 2019;28:2148-54.

30. Kumar S, Doughty C, Doros G, Selim M, Lahoti S, Gokhale S, et al. Recovery of swallowing after dysphagic stroke: an analysis of prognostic factors. J Stroke Cerebrovasc Dis 2014;23:56-62. 